\title{
Eradication of an epidemic methicillin-resistant Staphylococcus aureus (MRSA) from a geriatric university hospital: evidence from a 10-year follow-up
}

\author{
D. Mertz $\cdot$ R. Frei $\cdot$ N. Periat $\cdot$ C. Scheidegger • \\ M. Battegay $\cdot$ W. Seiler • A. F. Widmer
}

Received: 19 March 2010 / Accepted: 30 April 2010 / Published online: 3 June 2010

(C) Springer-Verlag 2010

\begin{abstract}
We report on a successful eradication of methicillin-resistant $S$. aureus (MRSA) after an epidemic in 1992 in the geriatric ward of a tertiary-care hospital. After identification of MRSA in seven patients, all patients and staff members in the geriatric ward underwent screening. A multifaceted intervention plan was implemented: contact isolation, optimization of infection control and decolonization of all MRSA carriers. Thirty-two patients and five staff members were found to be MRSA carriers. Twenty one of $32(66 \%)$ patients and all five staff members were successfully decolonized. Seven of 32 $(22 \%)$ patients died during the epidemic before decoloni-
\end{abstract}

\author{
D. Mertz $\cdot$ N. Periat $\cdot$ C. Scheidegger $\cdot$ M. Battegay $\cdot$ \\ A. F. Widmer $(\square)$ \\ Division of Infectious Diseases \& Hospital Epidemiology, \\ University Hospital of Basel, \\ Petersgraben 4, \\ CH-4031 Basel, Switzerland \\ e-mail:widmera@uhbs.ch \\ D. Mertz \\ e-mail:mertzd@uhbs.ch \\ N. Periat \\ e-mail: nad.periat@gmx.ch \\ C. Scheidegger \\ e-mail: scheideggerclaude@bluewin.ch \\ M. Battegay \\ e-mail: battegaym@uhbs.ch \\ R. Frei \\ Microbiology Laboratory, University Hospital of Basel, \\ Basel, Switzerland \\ e-mail: freir@uhbs.ch \\ W. Seiler \\ Geriatric Hospitals, University Hospital of Basel, \\ Basel, Switzerland \\ e-mail: walter-o.seiler@unibas.ch
}

zation. A couple was discharged with persisting MRSA colonization and two individuals were lost to follow-up. The eradication of the epidemic clone was proven by systematic screenings in 1995 and 1997. Since then, the strain has no longer been identified in our institution, based on epidemiological surveillance and molecular typing of all MRSA strains obtained from any specimen. This study provides strong evidence that long-term eradication of an MRSA epidemic in a hospital is feasible, and endemicity of MRSA after an outbreak can be avoided. The successful bundle approach for eradication of MRSA during an epidemic is expensive, but the long-term benefits likely outweigh the initial heavy use of resources.

\section{Introduction}

Since the first description of an isolate of methicillinresistant Staphylococcus aureus (MRSA) by Jevons in the 1960s [1], MRSA has emerged as the most important and most common multiresistant pathogen worldwide [2,3] and has become more common than methicillin-susceptible $S$. aureus in emergency rooms [4]. Although MRSA is primarily a nosocomial pathogen, it has become endemic in the community as well. However, MRSA prevalence is under control in certain European countries, such as Switzerland, [5] the Netherlands [6] and Scandinavian countries [7]. They follow a 'search and destroy' strategy [6], and antibiotic use is lower than in high-prevalence countries [8]. This 'search and destroy' policy including several components of infection control has been largely abandoned by most countries [9] due to lack of resources or limited success [10]. This strategy is even more difficult to apply in a geriatric setting because contact isolation and strict infection control measures may lead to social 
disturbances and less contact to health-care workers [11]. Furthermore, transmission rate in this setting is low [12]. Therefore, most geriatric units restrict contact isolation to MRSA patients with serious infections. Once being endemic, eradication of MRSA in the affected institution is regarded as not feasible.

Before implementation of a strict 'search and destroy" policy in our hospital in 1994, a MRSA epidemic in the geriatric ward of the University Hospital Basel took place starting in 1991. We provide strong evidence that an epidemic clone of MRSA can be definitely eradicated even in a geriatric ward. Only a few reports on successful eradication of MRSA in hospitals [13, 14] or long-term health care facilities have been published $[15,16]$, with some reports showing only a partial success [17-19]. In contrast to other reports, we have identified the MRSA clone and we provide a longer than 10-year follow-up with no recurrence of the epidemic clone.

\section{Patients, materials and methods}

\section{Setting}

The University Hospital Basel in Switzerland, neighboring France and Germany, is a tertiary-care university hospital with approximately 780 beds and 27,000 admissions yearly. In 1991, the geriatric ward was located in a separate building with 142 beds. The ward was divided into four floors (A-D).

\section{Epidemiologic work-up}

No formal surveillance was in place in 1991 and an infection control committee was created shortly before the epidemic. The epidemic was recognized after an increase in MRSA positive samples from the geriatric ward. The epidemiologic work-up included screening of all patients and staff and a multifaceted intervention plan, as described below in detail including: (i) contact isolation, (ii) routine decolonization of all MRSA patients, (iii) staff screening and (iv) furloughing, and decolonization of colonized health care workers (HCWs). Screening for MRSA always included a swab from the nares and the throat, a policy that later proved to be useful [5, 20]. All patients were followed-up after discharge until they became MRSA negative, died or refused further treatment.

Screening methods and microbiology

In addition to the routine swabs from the throat and the anterior nares, additional swabs were taken from several sites in the epidemic situation, i.e. the groin, perianal, rectum, urine, catheter insertion sites, and wounds. Repeat- ed screening of MRSA was begun $>48$ hours after completion of the decolonization treatment.

The swabs were cultured in a selective enrichment broth (brain heart infusion broth). Oxacillin resistance was tested using an oxacillin disc (National Committee for Clinical Laboratory Standards [21]) and more recently, a cefoxitin disc. In case of equivocal results, detection of aurease, penicillin-binding protein $2 \mathrm{a}$, and PCR for $m e c A$ gene and femA gene were performed. All MRSA strains isolated since the epidemic in 1992 underwent typing by pulsedfield gel electrophoresis (PFGE) [22]. Additionally, more than $90 \%$ of all subsequent strains underwent PCR-based [23] or spa-[22] typing. DNA sequences of spa-typing were analyzed by using Ridom StaphType software (Ridom GmbH, Wuerzburg, Germany) and synchronized using SpaServer (www.spaserver.ridom.de) [22]. PCR was used to detect selected virulence-associated genes [22].

\section{Intervention}

Infection control policies were enforced with a focus on teaching of correct hand hygiene with an alcoholic hand rub [24]. A dispenser for alcoholic hand rub was installed in front of every patient room and at the bedside of every patient. MRSA patients were put in contact isolation or were cohorted. Strict, monitored barrier precautions were implemented if working with MRSA patients, i.e. gloves, masks and gowns. All textiles with patient contact were changed daily and washed at a minimum temperature of $90^{\circ} \mathrm{C}$. Chlorhexidinealcohol replaced polyvinylpyrrolidone (PVP)-iodine for skin disinfection for its residual effect. A head nurse for the isolation ward was designated to enforce the new regulations. All HCWs from the isolation ward received additional training in infection control practices and weekly meetings allowed for exchange of information on microbiology results.

MRSA colonized HCWs were furloughed until having had a repeated negative screening for MRSA. All MRSA patients were transferred to one single isolation floor of the geriatric hospital: floor $\mathrm{C}$ on the upper level with 26 beds in 11 rooms. All other floors underwent terminal disinfection with $2 \%$ glutaraldehyde. The patients were classified in three different risk groups:

- Group A, high risk

Patients with MRSA colonization of wounds or respiratory tract infections were not allowed to leave their rooms. Full barrier precautions (gloves, gown and mask) were mandatory when entering a patients' room. - Group B, low risk

MRSA patients not meeting criteria for group A were allowed to leave their rooms for meals, and to socialize with other MRSA colonized patients. Full barrier precautions were mandatory when in contact 
Table 1 Scheme for decolonization

\begin{tabular}{|c|c|c|}
\hline Site of colonization & First choice & Application \\
\hline Skin & Soap containing $4 \%$ chlorhexidine & With every shower or bath \\
\hline Hands & Alcoholic hand rub & $\begin{array}{l}\text { Disinfection after nose cleaning and use of the rest rooms, before } \\
\text { preparation of meals and contact to other individuals }\end{array}$ \\
\hline Nose & $\begin{array}{l}\text { Mupirocin ointment (alternative: } \\
\text { fusidic acid) }\end{array}$ & Applied twice daily in both nares \\
\hline \multirow[t]{2}{*}{ Throat } & $\begin{array}{l}\text { Spray containing chlorhexidine } 0.2 \% \\
\text { (alternative: PVP-iodine) }\end{array}$ & Five sprays after every meal \\
\hline & $\begin{array}{l}\text { Chlorhexidine mouth rinse } 0.2 \% \\
\text { (alternative: PVP-iodine) }\end{array}$ & Gargle with $20 \mathrm{ml}$ after every meal \\
\hline $\begin{array}{l}\text { Gastrointestinal } \\
\text { (positive rectal swab) }\end{array}$ & $\begin{array}{l}\text { Vancomycin solution (alternative: } \\
\text { teicoplanin) }\end{array}$ & Vancomycin $0.5 \mathrm{~g}$ diluted in $10 \mathrm{ml}$ of water or coffee twice a day \\
\hline Perineal & $\begin{array}{l}\text { Mupirocin ointment (alternative: PVP- } \\
\text { iodine or bacitracinum) }\end{array}$ & Application using a swab after each defecation \\
\hline
\end{tabular}

PVP-iodine polyvinylpyrrolidone-iodine

with colonized sites. Gloves were indicated whenever patient contact was anticipated.

- Group C, transition group

Group C consisted of former group A or B patients, in whom three consecutive screenings of all body sites were negative after a completed decolonization scheme. These patients were transferred to the 'MRSA-free' or 'clean' part of the isolation ward, which was separated from the 'contaminated' part. This group was separated from the two former groups, and from non-colonized patients on the other floors. After two weeks, they were transferred to a 'clean' floor of the ward, if repeated cultures for MRSA once weekly remained negative.

Decolonization

All colonized patients and HCWs underwent decolonization using a draft version of a standardized decolonization treatment outlined in Table 1 [25]. Additionally, toothbrushes were disinfected daily in chlorhexidine $2 \%$. The skin was treated by bathing twice weekly and daily application of chlorhexidine soap (Hibiscrub ${ }^{\circledR}$ ). Chlorhexidine baths were continued every other week in nonMRSA carriers. In addition, patients with urinary tract colonization were treated systemically, e.g. using trimethoprim/sulfamethoxazole.

\section{Results}

Outbreak investigation

The first isolate of the epidemic was identified from a blood culture in May 1991 (patient 1). It took 18 months, until a second case (patient 2), hospitalized on the same ward (floor A) was identified in November 1992. Despite an

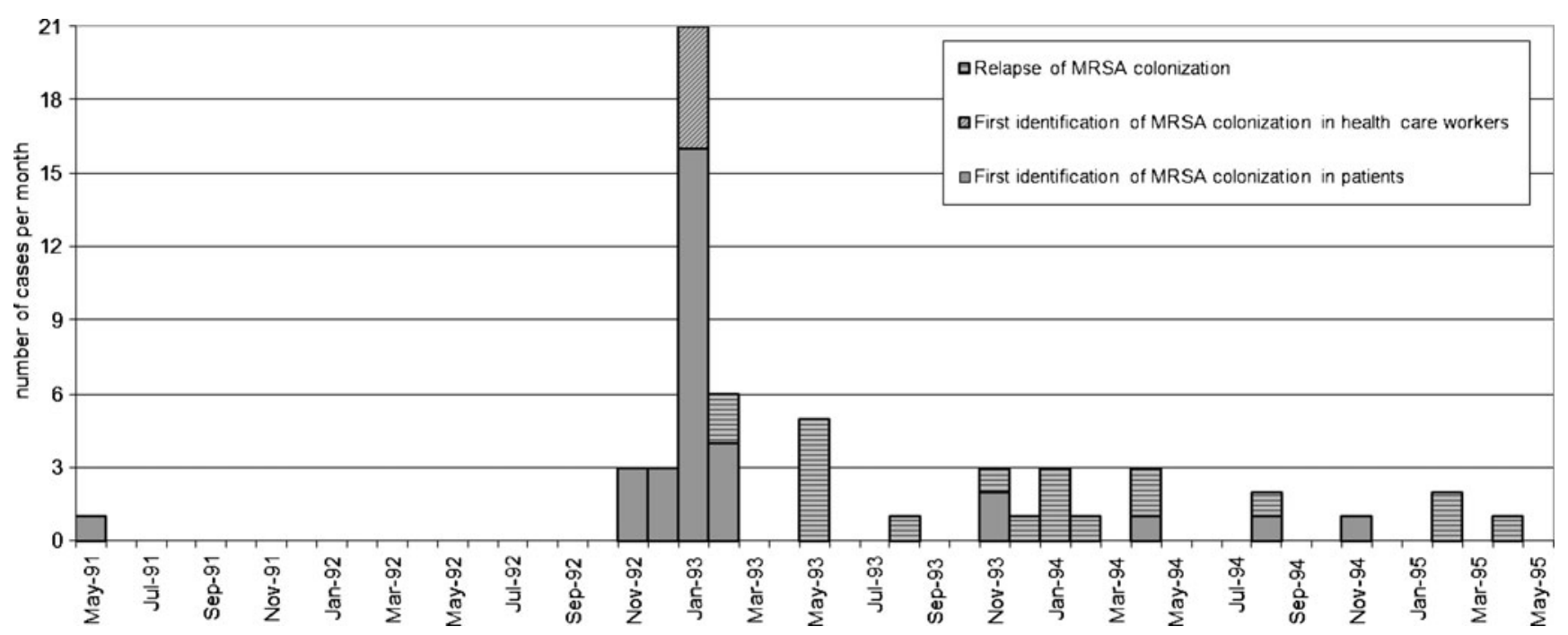

Fig. 1 Incidence of MRSA cases and relapses 
immediate isolation of the patient and screening of the roommate, two other patients (patients $3+4$ ) were identified on floor D. One of these patients was a former roommate of patient 2. Patient 5 on floor $\mathrm{C}$ and patient 6 on floor $\mathrm{B}$ followed. At that time, at least one MRSA carrier per floor was identified. In December 1992, PFGE confirmed an ongoing outbreak. In January 1993, all patients and staff members in the geriatric ward were screened for MRSA. The epidemic strain was identified in 16 additional patients. Furthermore, a total of three HCWs and two housekeeping personnel were detected as MRSA carriers.

The MRSA strain was resistant against aminoglycosides and quinolones, but susceptible for trimethoprim/sulfamethoxazole, doxycycline and fusidic acid. Molecular typing identified this type as PFGE type 1 and Spa-typing type t001. This Spa-type may be related to MLST type ST-5, ST222 or ST-228, all belonging to $\mathrm{CC} 5$, and is known as Southern German MRSA, Rhine Hesse MRSA, EMRSA-3, and New York clone (http://spaserver2.ridom.de/mlst.shtml) [26]. Genes for Panton-Valentine leukocidin, toxic-shock syndrome toxin, exfoliative toxin $\mathrm{A}, \mathrm{B}$ and $\mathrm{D}$ and staphylococcal enterotoxins A-E were not detected by PCR.

\section{Control of the epidemic}

In January 1993, the bundle of measures described above was strictly implemented. Nevertheless, four additional patients became MRSA carriers in the first month of the intervention, and many relapses in intermittently decolonized patients occurred (Fig. 1). The last patient newly diagnosed as a carrier of the epidemic MRSA strain was identified in November 1994.

The sites of colonization in the patients are summarized in Table 2. Of interest, urine was the second most common site of colonization (56\%), possibly due to the high prevalence of urinary incontinence (56\%) and urinary catheters in this population $(78 \%)$.

\section{Outcome}

Overall, the epidemic strain was identified in 32 patients, three HCWs and two housekeeping personnel between May 18, 1991 and May 29, 1995. The attack rate was 12.3\% during the peak of the epidemic.

Decolonization was successful in 21/32 (66\%) patients and $5 / 5(100 \%)$ staff members (three HCWs and two housekeeping personal). Before having had three sets of negative MRSA cultures, 7/32 (22\%) patients died. A couple was discharged as persistent MRSA carriers refusing any further therapy and two $(6 \%)$ patients were lost to follow-up. In the 2.5 years following the epidemic, i.e. 1993-1995, 20 reoccurrences were diagnosed in 14 patients. The mean duration of MRSA colonization was

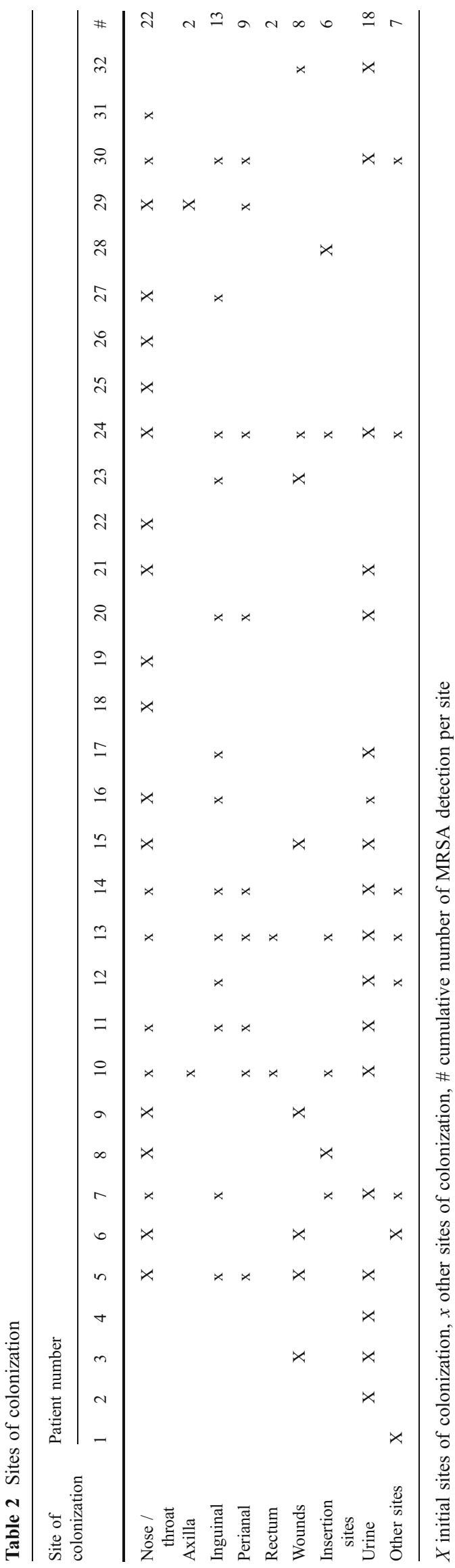


124.9 days (range 99.9-149.9). A mean of 1.6 (range 1-4) cycles of decolonization were performed until achievement of permanent MRSA negativity (Fig. 2).

During the two-year epidemic, an $18.3 \%$ increase in patient-days was observed. The additional costs for prolonged hospitalization of patients and the control measures were estimated to cost $\$ 195,000$ and $\$ 290,000$, respectively.

\section{Follow-up}

The epidemic MRSA strain was announced to be eradicated on May 29, 1995. All patients and staff of the geriatric ward were again screened in the nares and throat in August 1995 and June 1997. Not one single MRSA carrier was identified. Up to now, this MRSA type has no longer been observed, even when a representative strain of the outbreak was analyzed using additional molecular methods such as PCR [23] or spa typing [22].

\section{Discussion}

This is the first report of a successful eradication of an MRSA epidemic from a geriatric institution with long-term epidemiologic and molecular follow-up. Thirty-two patients and five staff members acquired the identical epidemic strain of MRSA, which was never again identified after cessation of the epidemic with a follow-up of longer than 10 years. The bundle approach led to the complete eradication of this epidemic strain from the hospital.

Not previously identified as a MRSA carrier, the index patient was transferred from a nursing home. He suffered from a serious form of an attention deficit hyperactivity syndrome and was walking around in the geriatric ward, which probably facilitated initial spread. Nevertheless, it took 18 months until an outbreak was recognized, likely explained by lack of surveillance and of an infection control team.

There are various guidelines describing how to control or prevent the epidemic spread of MRSA [27, 28], but few recommendations on how to eradicate MRSA from an institution in case of an epidemic. Once endemic, strains over time may vary, but MRSA eradication is considered as not feasible. Finnish authors reported eradication of an epidemic MRSA clone after outbreaks at the University Hospital of Turku [13] and in Helsinki [14] using a similar approach: screening of all patients and staff members, cohorting and strict isolation of all MRSA patients,

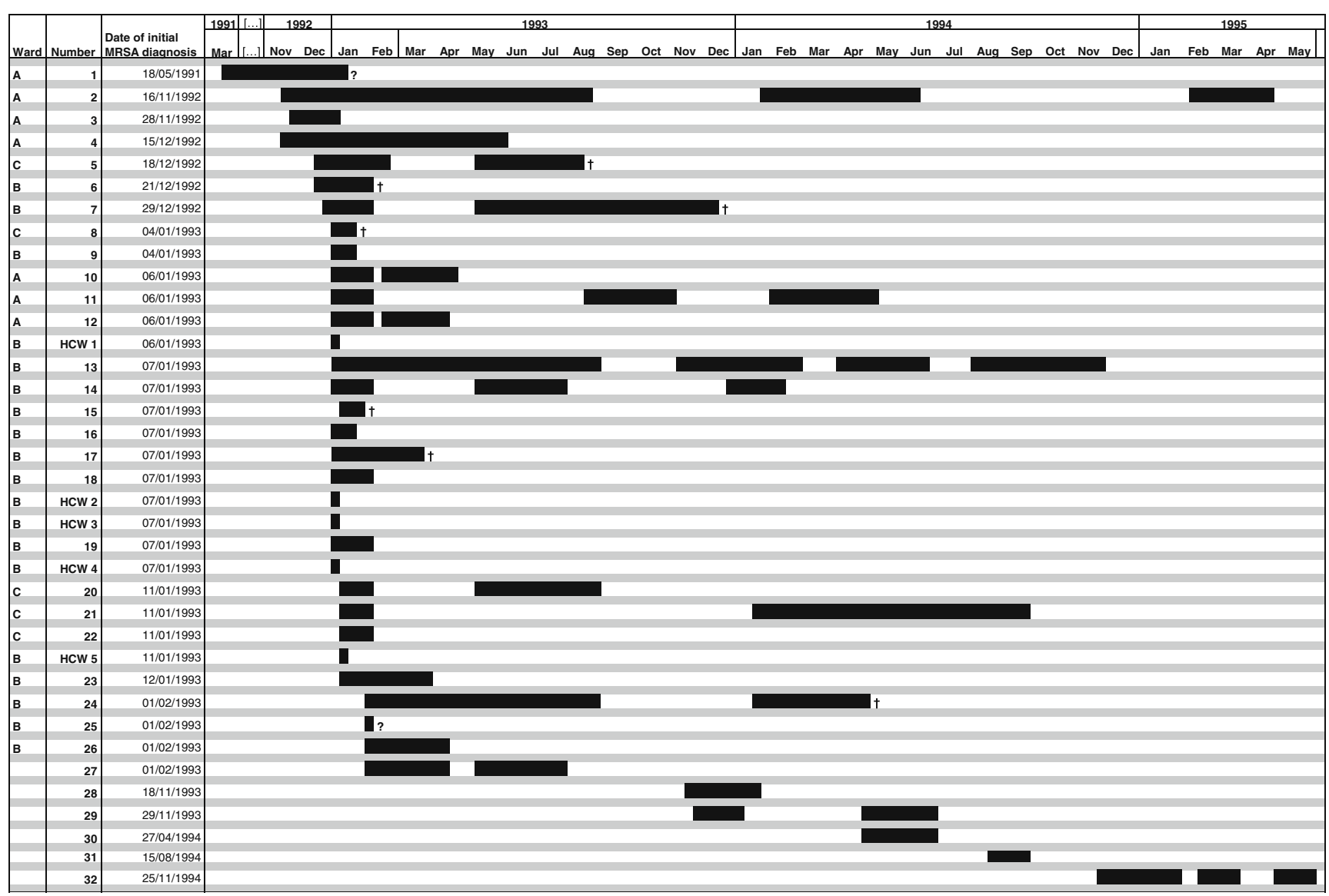

Fig. 2 Time line of MRSA colonization. $\%$ death, ? lost to follow-up, $H C W$ health care worker 
decolonization of all MRSA carriers and finally, teaching of an optimized hand disinfection. However, molecular typing was not completed, and the studies had a short period of follow-up. In addition, they did not actively screen the whole patient population after cessation of the outbreak and they did not perform additional throat screening, reducing the sensitivity of the screening $[5,20]$. All subsequent MRSA isolates from all clinics within the University Hospital Basel were molecularly characterized. To date, there is no evidence by epidemiological or molecular methods that this clone reoccurred in the University Hospital Basel, including in the geriatric ward which was moved in 2003 to another building. As geriatric hospitals and nursing homes send their samples to the university laboratory, the follow-up was as complete as possible. In contrast to the intervention in Turku, which involved other institutions in the surroundings as well, the epidemic in our hospital was controlled by an intervention limited to our hospital. As this approach was successful, we presume that the spread of MRSA in the community is negligible.

Because inter-hospital spread of epidemic and endemic MRSA strains are well known [29], the eradication of the MRSA epidemic was crucial to continuously control the MRSA prevalence in our low-endemic region. In contrast, an eradication of MRSA from hospitals in highly endemic countries seems impossible. We conclude that for future epidemics of resistant pathogens, e.g. vancomycin-nonsusceptible $S$. aureus, an early control using a 'search and destroy' strategy may be successful and that in the case of an epidemic, the measures presented in this report may eradicate the pathogen from the hospital before an endemic situation takes place. However, without the available rooms to isolate carriers, the budget - in our case a budget increase of almost $\$ 400,000$ - and the needed manpower, an eradication of MRSA would be difficult.

There are a few limitations to this report. Due to the long follow-up, part of the data was not available. The death of seven patients may have biased the results towards success. The outbreak was caused by a strain exhibiting phenotypic and molecular traits of a 'classical' hospitalonset MRSA strain. It remains to be demonstrated that eradication of a community-onset MRSA strain will be successful as well.

However, our report has several strengths. The long follow-up of more than 10 years and continued typing of all MRSA isolates prove that the epidemic strain was definitely eradicated and that there is no evidence that the strain responsible for the outbreak was replaced by another epidemic strain. Not one single case of MRSA colonization was detected in two full-house screenings of all patients and staff members in the geriatric ward after cessation of the epidemic and in routine post-discharge screenings of patients including home visits. Furthermore, we performed throat swabs in all patients which significantly increase the sensitivity of screening $[5,20]$.

Worldwide, the epidemic clone represents about $1.45 \%$ of all S. aureus strains; in the years 2009 and 2010, it was found most often in Germany, but also in other countries including Austria and Switzerland (http://spaserver2.ridom. de/spa-t001.shtml). However, in our hospital we found only three single patient isolates of this strain among 200 MRSA strains identified after the epidemic [22].

In conclusion, we show that in a low endemic region for MRSA, eradication of an MRSA epidemic in a hospital is feasible. Our experience may help to control epidemics due to other emerging pathogens, i.e. vancomycin-nonsusceptible $S$. aureus, and prevent these pathogens from becoming endemic in institutions.

Acknowledgments We thank Lukas Fenner, Microbiology Laboratory, University Hospital of Basel, for molecular characterization of the epidemic strain including spa-typing.

Previous presentation A part of the data was presented as an abstract at the 6th European Congress of Clinical Microbiology and Infectious Diseases (ECCMID) 1993, Seville, Spain, and an abstract was presented at the 19th European Congress of Clinical Microbiology and Infectious Diseases (ECCMID) 2009, Helsinki, Finland.

Funding This study was supported in part by grant 3200BO-104179 from the Swiss National Science Foundation.

Conflict of interest The authors declare that they have no conflict of interest.

\section{References}

1. Jevons MP, Coe AW, Parker MT (1963) Methicillin resistance in staphylococci. Lancet 1(7287):904-907

2. Klevens RM, Morrison MA, Nadle J, Petit S, Gershman K, Ray S, Harrison LH, Lynfield R, Dumyati G, Townes JM, Craig AS, Zell ER, Fosheim GE, McDougal LK, Carey RB, Fridkin SK (2007) Invasive methicillin-resistant Staphylococcus aureus infections in the United States. JAMA 298(15):1763-1771

3. Tiemersma EW, Bronzwaer SL, Lyytikainen O, Degener JE, Schrijnemakers P, Bruinsma N, Monen J, Witte W, Grundman H (2004) Methicillin-resistant Staphylococcus aureus in Europe, 1999-2002. Emerg Infect Dis 10(9):1627-1634

4. Moran GJ, Krishnadasan A, Gorwitz RJ, Fosheim GE, McDougal LK, Carey RB, Talan DA (2006) Methicillin-resistant S. aureus infections among patients in the emergency department. N Engl J Med 355(7):666-674

5. Mertz D, Frei R, Jaussi B, Tietz A, Stebler C, Fluckiger U, Widmer AF (2007) Throat swabs are necessary to reliably detect carriers of Staphylococcus aureus. Clin Infect Dis 45(4):475-477

6. Wertheim HF, Vos MC, Boelens HA, Voss A, VandenbrouckeGrauls CM, Meester MH, Kluytmans JA, van Keulen PH, Verbrugh HA (2004) Low prevalence of methicillin-resistant Staphylococcus aureus (MRSA) at hospital admission in the 
Netherlands: the value of search and destroy and restrictive antibiotic use. J Hosp Infect 56(4):321-325

7. Salmenlinna S, Lyytikainen O, Kotilainen P, Scotford R, Siren E, Vuopio-Varkila J (2000) Molecular epidemiology of methicillinresistant Staphylococcus aureus in Finland. Eur J Clin Microbiol Infect Dis 19(2):101-107

8. Goossens H, Ferech M, Vander Stichele R, Elseviers M (2005) Outpatient antibiotic use in Europe and association with resistance: a cross-national database study. Lancet 365(9459):579-587

9. Fluckiger U, Widmer AF (1999) Epidemiology of methicillinresistant Staphylococcus aureus. Chemotherapy 45(2):121-134

10. Harbarth S, Fankhauser C, Schrenzel J, Christenson J, Gervaz P, Bandiera-Clerc C, Renzi G, Vernaz N, Sax H, Pittet D (2008) Universal screening for methicillin-resistant Staphylococcus aure$u s$ at hospital admission and nosocomial infection in surgical patients. JAMA 299(10):1149-1157

11. Kirkland KB, Weinstein JM (1999) Adverse effects of contact isolation. Lancet 354(9185):1177-1178

12. Bradley SF (1999) Methicillin-resistant Staphylococcus aureus: long-term care concerns. Am J Med 106(5A):2S-10S, discussion $48 \mathrm{~S}-52 \mathrm{~S}$

13. Kotilainen P, Routamaa M, Peltonen R, Oksi J, Rintala E, Meurman O, Lehtonen OP, Eerola E, Salmenlinna S, VuopioVarkila J, Rossi T (2003) Elimination of epidemic methicillinresistant Staphylococcus aureus from a university hospital and district institutions, Finland. Emerg Infect Dis 9(2):169-175

14. Pastila S, Sammalkorpi KT, Vuopio-Varkila J, Kontiainen S, Ristola MA (2004) Control of methicillin-resistant Staphylococcus aureus outbreak involving several hospitals. J Hosp Infect 58 (3):180-186

15. Simor AE, Augustin A, Ng J, Betschel S, McArthur M (1994) Control of MRSA in a long-term care facility. Infect Control Hosp Epidemiol 15(2):69-70

16. Kotilainen P, Routamaa M, Peltonen R, Evesti P, Eerola E, Salmenlinna S, Vuopio-Varkila J, Rossi T (2001) Eradication of methicillin-resistant Staphylococcus aureus from a health center ward and associated nursing home. Arch Intern Med 161(6):859863

17. Cederna JE, Terpenning MS, Ensberg M, Bradley SF, Kauffman CA (1990) Staphylococcus aureus nasal colonization in a nursing home: eradication with mupirocin. Infect Control Hosp Epidemiol 11(1):13-16

18. Kauffman CA, Terpenning MS, He X, Zarins LT, Ramsey MA, Jorgensen KA, Sottile WS, Bradley SF (1993) Attempts to eradicate methicillin-resistant Staphylococcus aureus from a long-term-care facility with the use of mupirocin ointment. Am J Med 94(4):371-378
19. Strausbaugh LJ, Jacobson C, Sewell DL, Potter S, Ward TT (1992) Antimicrobial therapy for methicillin-resistant Staphylococcus aureus colonization in residents and staff of a Veterans Affairs nursing home care unit. Infect Control Hosp Epidemiol 13 (3):151-159

20. Mertz D, Frei R, Periat N, Zimmerli M, Battegay $M$, Fluckiger U, Widmer AF (2009) Exclusive Staphylococcus aureus throat carriage: at-risk populations. Arch Intern Med 169(2):172-178

21. National Committee for Clinical Laboratory Standards (1990) Performance standards for antimicrobial disc susceptibility tests. Approved standard M2-A4 (plus updates and informational supplements). National Committee for Clinical Laboratory Standards, Villanova, PA

22. Fenner L, Widmer AF, Dangel M, Frei R (2008) Distribution of spa types among methicillin-resistant Staphylococcus aureus isolates during a 6 year period at a low-prevalence University Hospital. J Med Microbiol 57(Pt 5):612-616

23. Stranden A, Frei R, Widmer AF (2003) Molecular typing of methicillin-resistant Staphylococcus aureus: can PCR replace pulsed-field gel electrophoresis? J Clin Microbiol 41(7):31813186

24. Widmer AF, Conzelmann M, Tomic M, Frei R, Stranden AM (2007) Introducing alcohol-based hand rub for hand hygiene: the critical need for training. Infect Control Hosp Epidemiol 28 (1):50-54

25. Buehlmann M, Frei R, Fenner L, Dangel M, Fluckiger U, Widmer AF (2008) Highly effective regimen for decolonization of methicillin-resistant Staphylococcus aureus carriers. Infect Control Hosp Epidemiol 29(6):510-516

26. Strommenger B, Braulke C, Heuck D, Schmidt C, Pasemann B, Nubel U, Witte W (2008) Spa typing of Staphylococcus aureus as a frontline tool in epidemiological typing. J Clin Microbiol 46 (2):574-581

27. Loveday HP, Pellowe CM, Jones SR, Pratt RJ (2006) A systematic review of the evidence for interventions for the prevention and control of methicillin-resistant Staphylococcus aureus (1996-2004): report to the joint MRSA working party (subgroup A). J Hosp Infect 63(Suppl 1):S45-S70

28. Muto CA, Jernigan JA, Ostrowsky BE, Richet HM, Jarvis WR, Boyce JM, Farr BM (2003) SHEA guideline for preventing nosocomial transmission of multidrug-resistant strains of Staphylococcus aureus and enterococcus. Infect Control Hosp Epidemiol 24(5):362-386

29. Casewell MW (1995) New threats to the control of methicillinresistant Staphylococcus aureus. J Hosp Infect 30(Suppl):465471 